\title{
von Willebrand Factor Antigen Predicts Outcomes in Patients after Liver Resection of Hepatocellular Carcinoma
}

\author{
Christoph Schwarz ${ }^{1}$, Fabian Fitschek ${ }^{1}$, Martina Mittlböck ${ }^{2}$, Veronika Saukel ${ }^{1}$, Simona Bota $^{3}$, Monika Ferlitsch ${ }^{2}$, \\ Arnulf Ferlitsch ${ }^{4}$, Martin Bodingbauer ${ }^{1}$, and Klaus Kaczirek ${ }^{1}$ \\ ${ }^{I}$ Department of General Surgery and ${ }^{2}$ Center for Medical Statistics, Informatics, and Intelligent Systems, Section for Clinical Biometrics, \\ Medical University Vienna, Vienna, ${ }^{3}$ Department of Gastroenterology, Hepatology, Nephrology and Endocrinology, Klinikum Klagenfurt am \\ Wörthersee, Klagenfurt, and ${ }^{4}$ Division of Gastroenterology and Hepatology, Department of Internal Medicine III, Medical University Vienna, \\ Vienna, Austria
}

Background/Aims: von Willebrand factor antigen (vWF-Ag) is a noninvasive predictor of portal hypertension that serves as a negative prognostic marker in various malignancies. Increased portal hypertension is associated with higher postoperative morbidity and decreased survival after hepatectomy. The purpose of this study was to determine the correlation between vWF-Ag, postoperative morbidity and oncological outcome. Methods: This analysis includes 55 patients who underwent liver resection for hepatocellular carcinoma (HCC) between 2008 and 2015 with available preoperative vWF-Ag levels. The primary endpoints were postoperative complications and long-term outcome, including overall and diseasefree survival. Results: The median plasma level of vWF-Ag was $191 \%$ (range, $162.5 \%$ to $277 \%$ ). There was a significant correlation between vWF-Ag levels and tumor size in the resected specimens $(p=0.010, r=0.350)$. Patients who developed any grade of postoperative complication had significantly higher preoperative vWF-Ag levels (216\% [range, 178\% to $283.25 \%$ ] vs $176 \%$ [range, $148 \%$ to $246 \%$ ], $p=0.041$ ). Median overall survival was 39.8 months in patients with high vWF-Ag levels ( $\geq 191 \%$ ) compared with 73.4 months in patients with low levels $(<191 \%, p=0.007)$. Of note, there was a remarkable disparity in the number of patients who died of HCC with low versus high vWF-Ag levels (14.8\% vs $28.6 \%$, $p=0.011$ ). Conclusions: vWF-Ag may serve as a prognostic marker for the outcome of patients undergoing liver resection for HCC that is closely connected to tumor size, postoperative complication rate and long-term outcome. (Gut Liver 2020;14:218-224)

Key Words: von Willebrand factor; Outcome; Hepatectomy;
Carcinoma, hepatocellular

\section{INTRODUCTION}

Hepatocellular carcinoma (HCC) is the third most cause of cancer-related death and its incidence and mortality is increasing. ${ }^{1-4}$ Liver resection is the first-line treatment for patients with solitary HCC, however as in most cases HCC develops in an established liver disease, ${ }^{2}$ resection is only recommended in patients with well-preserved liver function, defined as normal bilirubin with either hepatic venous pressure gradient (HVPG) $\leq 10 \mathrm{~mm} \mathrm{Hg}$ or platelet count $\geq 100,000 / \mathrm{mL}{ }^{5}$ Even though outcome of liver resection has improved significantly over the last decades, ${ }^{6}$ patients still face a considerable morbidity and mortality, ${ }^{7}$ thus prognostic parameters to determine outcome are urgently warranted.

It has been shown that von Willebrand factor antigen (vWF$\mathrm{Ag}$ ) is a simple and noninvasive predictor of clinically significant portal hypertension (CSPH; HVPG $\geq 10 \mathrm{~mm} \mathrm{Hg}$ ), that predicts survival and decompensation in patients with liver cirrhosis, independently of Child-Pugh and Model for End-Stage Liver Disease (MELD) score. ${ }^{8}$ Additionally vWF plays a role in platelet-tumor cells interactions, angiogenesis and apoptosis. ${ }^{9}$ Several studies have shown elevated plasma vWF-Ag levels in both hematologic and non-hematologic malignancies. ${ }^{10-12}$ Thus vWF-Ag may also serve as a negative prognostic marker for neoplastic disorders.

The aim of the study was to investigate the association between preoperatively measured vWF-Ag levels with postoperative morbidity and oncological outcome defined as disease-free survival (DFS) and overall survival (OS) in patients undergoing

Correspondence to: Martin Bodingbauer

Department of General Surgery, Medical University Vienna, Waerhingergürtel 18-20, Vienna 1090, Austria

Tel: +43-1-40400-56210, Fax: +43-1-40400-69, E-mail: martin.bodingbauer@meduniwien.ac.at

Received on March 8, 2017. Revised on October 26, 2017. Accepted on November 25, 2017. Published online November $12,2018$. pISSN 1976-2283 eISSN 2005-1212 https://doi.org/10.5009/gnl17115

(c) This is an Open Access article distributed under the terms of the Creative Commons Attribution Non-Commercial License (http://creativecommons.org/licenses/by-nc/4.0) which permits unrestricted non-commercial use, distribution, and reproduction in any medium, provided the original work is properly cited. 
hepatectomy for HCC.

\section{MATERIALS AND METHODS}

\section{Patients}

This is a retrospective study including all patients undergoing primary liver resection for HCC with curative intent between 2008 and 2015, who were classified Child-Pugh A and had preoperative vWF-Ag, indocyanine green (ICG)-clearance and HVPG measurements as part of their routine work-up. All patients underwent staging computer tomography of the liver, abdomen and thorax before surgery. Additional magnetic resonance imaging of the liver was performed in most patients. Patients were followed routinely at least every 6 months at the outpatient clinic of the Department of General Surgery, Medical University of Vienna. The study was approved by the Ethics Committee of the Medical University of Vienna and was in in accordance with the Declaration of Helsinki and the International Conference on Harmonization of Technical Requirements for Registration of Pharmaceuticals for Human Use Harmonized Tripartite guideline for good clinical practice (IRB number: 1603/2013). Written informed consent was waived.

\section{Serological tests}

For all patients routine serological tests were performed before surgery: hematology, biochemistry (bilirubin, albumin, creatinine, aminotransferases, albumin, alkaline phosphatase, $\gamma$ glutamyl-transpeptidase), coagulation parameters (prothrombin time, INR), $\alpha$-fetoprotein (AFP) and C-reactive protein (CRP).

Plasma levels of vWF-Ag and factor VIII were measured as described previously ${ }^{13}$ using a fully automated STA analyzer and vWF-Liatest (Diagnostica Stago, Paris, France). According to their vWF-Ag levels patients were grouped in a high group separated by the median (191\%).

ICG plasma disappearance rate (ICG-PDR) and ICG retention rate at 15 minutes (ICG-R15) were measured noninvasively on the day before liver resection by digital pulse densitometry using a finger piece sensor connected to a LiMON device (Pulsion Medical Systems, Munich, Germany) as described earlier. ${ }^{14}$ ICGPDR values of $>18 \% / \mathrm{min}$ and ICG-R15 values of $<10 \%$ were assumed to be normal. ${ }^{15}$

\section{HVPG measurements}

Portal pressure was evaluated by means of HVPG according to international standards as previously described. ${ }^{16,17}$ Briefly, a balloon occlusion catheter (7 F, Ferlitsch HVPG Catheter; Pejcl Medizintechnik, Austria) was placed into a large liver vein and mean HVPG was calculated from a minimum of three measurements of free and wedged hepatic vein pressures.

\section{Liver resection}

Liver resections were performed by an experienced team in a two-surgeon technique using Cavitron ultrasonic surgical aspirator (CUSA ${ }^{\mathrm{TM}}$; Valleylab, Boulder, CO, USA) and bipolar forceps. Temporary inflow occlusion was applied in a minority of patients. Resections were classified according to the International Hepato-Pancreato-Biliary Association Brisbane 2000 nomenclature. ${ }^{18}$ Minor resections comprised less than three and major resections more than three Couinaud segments. Any postoperative morbidity was recorded within the first 30 days following liver resection. Morbidity was defined as any deviation from the normal postoperative course and the grade was classified according to the Clavien-Dindo classification. ${ }^{19}$

\section{Histopathology}

Tumor size, Edmondson and Steiner grade, ${ }^{20}$ and tumor stage according to the seventh edition of the International Union Against Cancer (UICC 2009) classification of malignant tumors were assessed. Liver fibrosis was classified as proposed by Batts and Ludwig. ${ }^{21}$

\section{Statistical analysis}

Statistical analysis was performed using GraphPad Prism, version 6 (GraphPad Prism Software MedCalc Software ${ }^{\circledR}$, La Jolla, CA, USA). Continuous variables were reported as median and interquartile range (IQR1-IQR3) and categorical variables were reported as absolute numbers and percentages. Student t-test was used for group comparisons of continuous variables with normal distribution or with the Mann-Whitney U-test otherwise. Categorical values were compared with a chi-square test or in case of sparse data with a Fisher-exact test. Ordinal variables were tested by a chi-square trend test. Associations between continuous variables were assessed by Pearson correlation coefficients, where right-skew variables were transformed by natural logarithm to achieve normally distributed variables. As one patient has died during hospital stay, a longer hospital stay in days (concretely 130 days) was imputed for this patient in order to represent death as worst outcome (worse than any length of hospital stay). Furthermore a nonparametric Spearman correlation $\left(\mathrm{r}_{\mathrm{s}}\right)$ was used to properly address the ordered outcome.

Survival was calculated using a Kaplan-Meier analysis and comparison was performed using a log-rank test between groups. Group differences were also described by hazard ratios (HR) and corresponding 95\% confidence intervals (CI) estimated by Cox regression. Additionally, the effect of vWF on recurrence free and OS were estimated by a multiple Cox regression model to adjust for other relevant prognostic variables.

All $p$-values are two-sided and $p<0.05$ was considered statistically significant.

\section{RESULTS}

\section{Patients' characteristics}

Overall, 55 patients were included in this analysis. The me- 
Table 1. Patient Characteristics

\begin{tabular}{|c|c|c|c|c|}
\hline Characteristic & Overall $(n=55)$ & vWF-Ag <191 (n=27) & vWF-Ag $\geq 191(n=28)$ & p-value \\
\hline Male sex & 48 (87.3) & $24(88.9)$ & $24(85.7)$ & 1.000 \\
\hline Age, yr & $67.1(62.7-71.7)$ & $65.1(61.6-70.1)$ & $69.6(63.2-74.1)$ & 0.165 \\
\hline BMI, $\mathrm{kg} / \mathrm{m}^{2}$ & $28.3(25.8-31.1)$ & $27.3(24.4-29)$ & $29(27-32.4)$ & 0.486 \\
\hline Etiology of liver disease & & & & 0.695 \\
\hline Viral & $15(27.3)$ & $8(30.8)$ & 7 (25.9) & \\
\hline Non-viral & $38(69.1)$ & $18(69.2)$ & $20(74.1)$ & \\
\hline Not determined ${ }^{*}$ & $2(3.6)$ & 1 & 1 & \\
\hline \multicolumn{5}{|l|}{ Comorbidities } \\
\hline IDDM & $5(9.1)$ & $3(11.1)$ & $2(7.1)$ & 0.670 \\
\hline Adipositas & $14(25.5)$ & $5(18.5)$ & $9(32.1)$ & 0.355 \\
\hline Arterial hypertension & $33(60.0)$ & $15(55.6)$ & $18(64.3)$ & 0.588 \\
\hline No comorbidities & $6(10.1)$ & $3(11.1)$ & $3(10.7)$ & 1.000 \\
\hline Portal vein embolisation & $8(14.5)$ & $4(14.8)$ & $4(14.3)$ & 1.000 \\
\hline \multicolumn{5}{|l|}{ ICG clearance } \\
\hline PDR & $17(14.6-21.3)$ & $19.3(16.7-25)$ & $15(13.1-17.8)$ & 0.250 \\
\hline R15 & $7.5(4.3-13.1)$ & $5.6(2.5-7.9)$ & $11(6.3-14)$ & 0.023 \\
\hline Thrombocytes, G/ $/ \mu \mathrm{L}$ & 197 (154-234) & 199 (157.5-222.5) & $188.5(149.5-244.5)$ & 0.556 \\
\hline$\alpha$-Fetoprotein, ng/mL & $5.9(3-133.5)$ & $9.9(2.8-150.6)$ & $5.7(3.2-11)$ & 0.706 \\
\hline HVPG, mm Hg & $5(4-8.25)$ & $5(4-5)$ & $6(4-10)$ & 0.080 \\
\hline WHPG, mm Hg & $14(10-16)$ & $11(9-15)$ & $15(14-19)$ & 0.017 \\
\hline Grade of fibrosis & & & & 0.306 \\
\hline I & $10(18.2)$ & $3(11.1)$ & $7(25.0)$ & \\
\hline II & $8(14.5)$ & $5(18.5)$ & $3(10.7)$ & \\
\hline III & $5(9.1)$ & $2(7.4)$ & $3(10.7)$ & \\
\hline IV & $25(45.5)$ & $11(40.7)$ & $14(50.0)$ & \\
\hline Type of resection, & & & & 0.163 \\
\hline Major hepatectomy & $20(36.4)$ & 7 (25.9) & $13(46.4)$ & \\
\hline Minor hepatectomy & $35(63.6)$ & $20(74.1)$ & $15(53.6)$ & \\
\hline Tumor size, cm & $5.25(3.25-7.75)$ & $5.3(3.6-6.5)$ & $5.5(3-11.8)$ & 0.993 \\
\hline Clavien-Dindo score & & & & 0.078 \\
\hline I & $5(9.1)$ & $4(14.8)$ & $1(3.6)$ & \\
\hline II & 7 (12.7) & $1(3.7)$ & $6(21.4)$ & \\
\hline IIIa & $4(7.3)$ & $1(3.7)$ & $3(10.7)$ & \\
\hline $\mathrm{IIIb}$ & $3(5.5)$ & $2(7.4)$ & $1(3.6)$ & \\
\hline IV & $2(3.6)$ & 0 & $2(7.1)$ & \\
\hline $\mathrm{V}$ & $1(1.8)$ & 0 & $1(3.6)$ & \\
\hline Length of stay, day ${ }^{\dagger}$ & $10(8-17.5)$ & $8(8-13.5)$ & $11(9-28.8)$ & 0.008 \\
\hline
\end{tabular}

Data are presented as number (\%) or median (interquartile range).

vWF-Ag, von Willebrand factor antigen; BMI, body mass index; IDDM, insulin-dependent diabetes mellitus; NIDDM, non-IDDM; ICG, indocyanine green; PDR, plasma disappearance rate; R15, retention rate after 15 minutes; HVPG, hepatic venous pressure gradient; WHPG, wedged hepatic venous pressure.

*Patients whose values were not determined were not included in the analysis; ${ }^{\dagger}$ In case of death before discharge, length of stay was imputed with 130. 
dian age was 67.1 years (range, 62.7 to 71.7 years) and all patients were Child-Pugh class A (100\%). The type of liver resection was minor hepatectomy in most cases (63.6\%). Further baseline characteristics are shown in Table 1. Median follow-up was 20.3 months following liver resection.

\section{2. vWF-Ag levels}

Overall, the median plasma level of vWF-Ag was 191\% (range, $162.5 \%$ to $277 \%$ ). There was no correlation between log-HVPG and log-vWF-Ag levels measured before liver resection $(\mathrm{p}=0.256$, $\mathrm{r}=0.167$ based on ln-transformation of HVPG and vWF-Ag). However, we found moderate but significant correlations between log-vWF-Ag and log-tumor size (largest diameter)

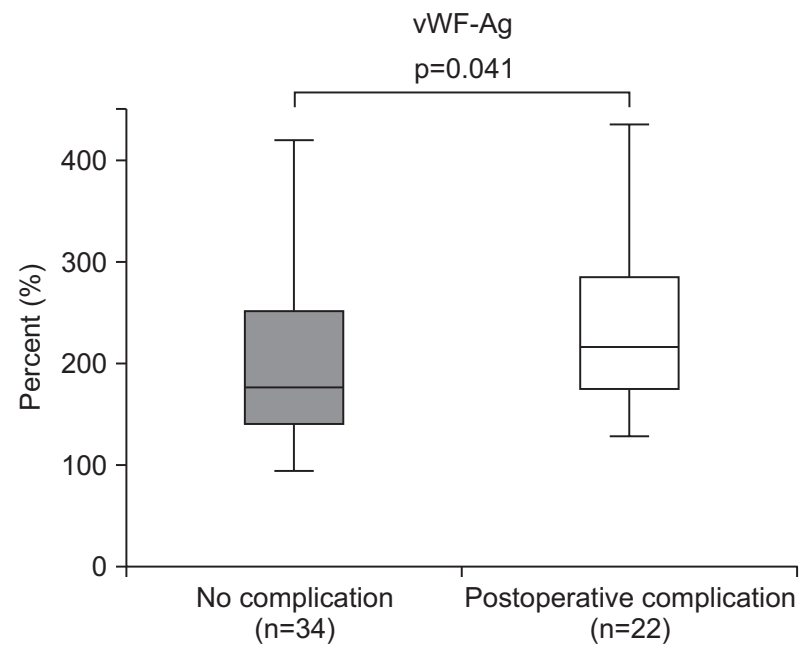

Fig. 1. von Willebrand factor antigen (vWF-Ag) levels were higher in patients with any grade of postoperative complication compared with patients without postoperative complications $(\mathrm{p}=0.041)$.

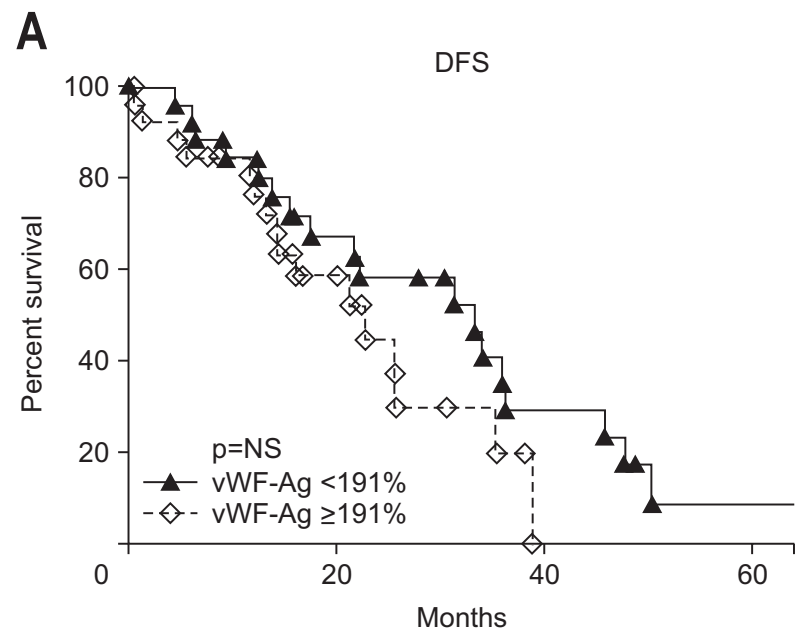

( $\mathrm{p}=0.010, \mathrm{r}=0.350$ for log-transformed vWF-Ag and tumor size) wedged hepatic venous pressure $(p=0.019, r=0.344)$ and length of stay ( $\left.\mathrm{p}=0.0003, \mathrm{r}_{\mathrm{s}}=0.465\right)$.

\section{Outcome after liver resection for $\mathrm{HCC}$ resection}

Any degree of postoperative morbidity was observed in 22 patients (40\%). According to Clavien-Dindo, five patients had grade I complications (9.1\%), seven patients grade II (12.7\%), four patients grade IIII (7.3\%), three patients IIIlb (5.5\%), two patients grade IV (3.6\%) and one patient suffered from a grade $\mathrm{V}$ complication (1.8\%).

vWF-Ag levels were significantly elevated in patients who experienced any grade of postoperative morbidity (216\% [178\% to $283.25 \%$ ] vs $176 \%$ [ $148 \%$ to $246 \%$ ], $\mathrm{p}=0.041$ ) (Fig. 1). Moreover, by using a cutoff value at the median of 191\%, vWF-Ag was highly prognostic for outcome following liver resection for HCC. There was a trend towards more, and more severe surgical complications in patients with a vWF-Ag level of more than $191 \%$. In line with this, length of stay was significantly prolonged in the latter group ( 8 days [range, 8 to 13.5 days] vs 11 days [range, 9 to 28.8 days], $\mathrm{p}=0.008$ ) (Table 1).

Regarding long-term outcome, there was a trend towards a shorter DFS in patients with a high vWF-Ag level (median DFS, 22.8 months vs 33.4 months, $\mathrm{p}=0.161$; HR, 1.8; 95\% CI, 0.8 to 3.7) (Fig. 2A). OS was significantly diminished in patients with elevated vWF-Ag levels (median survival time, 39.8 months vs 73.4 months, $\mathrm{p}=0.007$; HR, 4.06; 95\% CI, 1.4 to 12.1) (Fig. 2B). Of note eight of 28 patients (28.6\%) from the high vWF-Ag group and four of $27(14.8 \%)$ died of HCC $(\mathrm{p}=0.011)$.

In a multivariate survival analysis differences between vWF$\mathrm{Ag}$ above and below median was adjusted for other important prognostic factors (Table 2). The hazard ratio for vWF-Ag in-

\section{B}

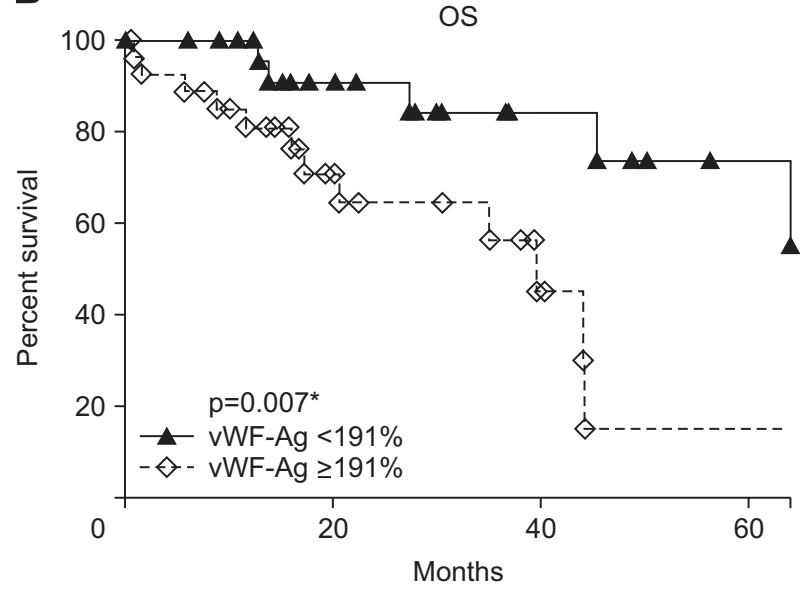

Fig. 2. Prognostic impact of preoperatively measured von Willebrand factor antigen (vWF-Ag) levels on long-term outcome after hepatectomy for hepatocellular carcinoma. (A) There was a trend towards reduced disease-free survival (DFS) in patients with a vWF-Ag level $\geq 191 \%$ (median DFS, 22.8 mo vs 33.4 mo, p=NS). (B) Overall survival (OS) was significantly reduced in patients with elevated vWF-Ag levels (39.8 mo vs 73.4 mo, $\mathrm{p}=0.007$ ).

NS, not significant. ${ }^{*} \mathrm{p}<0.05$. 
Table 2. Factors Influencing DFS and OS

\begin{tabular}{|c|c|c|}
\hline Parameter & HR (95\% CI) & $\mathrm{p}$-value \\
\hline \multicolumn{3}{|l|}{ DFS } \\
\hline vWF-Ag* & $2.31(1.04-5.15)$ & 0.041 \\
\hline Vascular infiltration $^{\dagger}$ & $1.23(0.55-2.74)$ & 0.613 \\
\hline Grade of fibrosis $^{\ddagger}$ & $0.79(0.61-1.03)$ & 0.080 \\
\hline Age, yr & $0.95(0.92-0.99)$ & 0.005 \\
\hline Tumor size, cm & $1.09(1.00-1.19)$ & 0.053 \\
\hline \multicolumn{3}{|l|}{ OS } \\
\hline vWF-Ag* & 4.89 (1.28-18.69) & 0.020 \\
\hline Vascular infiltration $^{\dagger}$ & $0.94(0.29-3.01)$ & 0.919 \\
\hline Grade of fibrosis $^{\ddagger}$ & $0.91(0.60-1.39)$ & 0.663 \\
\hline Age, yr & $1.03(0.96-1.10)$ & 0.436 \\
\hline Tumor size, cm & $1.09(0.98-1.22)$ & 0.118 \\
\hline
\end{tabular}

DFS, disease-free survival; OS, overall survival; HR, hazard ratio; CI, confidence interval; vWF-Ag, von Willebrand factor antigen.

*Reference level: vWF-Ag < 191\%; ${ }^{\dagger}$ Reference level: no vascular infiltration; ${ }^{\ddagger}$ Grade 0 to IV.

creased for DFS (HR, 2.31; 95\% CI, 1.04 to 5.15; $p=0.041$ ) after adjusting for effects of other covariates. Of note, patient's age (HR, 0.95; 95\% CI, 0.92 to 0.99; $p=0.005$ ) was also significantly associated with DFS. Adjusted HR of vWF-Ag for OS also slightly increased (HR, 4.89; 95\% CI, 1.28 to $18.69 ; \mathrm{p}=0.020$ ) (Table 2).

To test the influence of the underlying liver disease on the prediction of vWF-Ag we divided the patients according to the grade of fibrosis in low grade patients (F0-F2) and patients with fibrosis/cirrhosis (F3, F4) (Supplementary Fig. 1). Interestingly, vWF-Ag was especially predictive in patients with no or only mild signs of fibrosis in the resected specimen.

\section{DISCUSSION}

Current European Association for the Study of the Liver (EASL) guidelines base treatment allocation of patients with HCC on the Barcelona-Clinic Liver Cancer (BCLC) staging system. ${ }^{5,22,23}$ Resection is recommended for BCLC stages 0 and A with single tumors in case of normal bilirubin and portal pressure. Gold standard for the evaluation of portal pressure is measurement of HVPG. ${ }^{824,25}$ However, this is an invasive method and not universally available, even in specialized centers. vWF-Ag is a new, simple and noninvasive predictor of CSPH and can predict survival and decompensation in liver cirrhosis independently of MELD score. To the best of our knowledge, this is the first study that analyzed its ability to predict postoperative outcome in patients undergoing liver resection for HCC.

It has been shown that portal pressure correlates with survival after hepatectomy ${ }^{26,27}$ and that vWF-Ag correlates with HVPG values in patients with cirrhosis, ${ }^{28-30}$ thus we have speculated to find a connection between vWF-Ag levels and HVPG measured before liver resection. Unexpectedly, we could not find any significant connection between HVPG and vWF-Ag levels in our study cohort. We assume that the lack of correlation is a result of the exclusion of patients with high HVPG levels and impaired liver function for resection.

Postoperative complications were observed in $40 \%$, which is in range of reported rates in the literature. ${ }^{31}$ Patients with any grade of postoperative complication had an increased level of vWF-Ag. Inversely, patients with a vWF-Ag level of more than $191 \%$ had a trend towards more and more severe postoperative morbidity and had a significantly prolonged length of stay.

vWF not only plays a critical role in hemostasis but has been suggested to potentially promote tumor growth and dissemination. $^{9}$ While experimental studies suggest that vWF may reduce the formation of metastases due to an anti-angiogenic and pro-apoptotic potential, ${ }^{9}$ clinical studies show a link between increased plasma vWF-Ag levels and worse prognosis and advanced stage in colorectal, ${ }^{32,33}$ gastric, ${ }^{11}$ urinary bladder ${ }^{34}$ and ovarian cancer. ${ }^{35}$ It has been suggested that vWF may promote tumor progression, angiogenesis and metastasis formation via endothelial cell activation. ${ }^{36,37}$ In our study, vWF-Ag levels were significantly correlated with tumor size indicating a more advanced stage of disease. Of note, no correlation was found between tumor size and AFP. Furthermore, there was a trend towards a shorter DFS even though the observed difference did not reach statistical significance. These results suggest that vWF-Ag might also serve as a tumor marker that should be validated in future studies.

Patients with elevated vWF-Ag levels had a significantly reduced OS compared to patients with low levels. Moreover, the proportion of patients who died of HCC were doubled in patients with vWF-Ag levels of more than 191\% compared to patients with lower levels, suggesting a prognostic role of vWF$\mathrm{Ag}$ in oncological outcome. Whether vWF plays a causative role or just reflects an epiphenomenon of the underlying cancerassociated inflammatory process or other pathophysiologic changes has not yet been elucidated. ${ }^{9}$

There are several limitations that need to be regarded when interpreting the results. Firstly, the study is of retrospective nature, which has a potential bias inherited to the study's design. Secondly, this analysis only includes patients with good liver function and low HVPG levels, thus assumptions to use vWF$\mathrm{Ag}$ to define operability cannot be made.

To sum up, vWF-Ag is an easy to measure, valuable tool to discriminate between patients with a high risk of postoperative morbidity and an impaired long-term outcome. Further prospective trials are warranted to confirm the prognostic value of vWF-Ag and to define its role for predicting morbidity in patients undergoing liver resection in the presence of portal hypertension. 


\section{CONFLICTS OF INTEREST}

No potential conflict of interest relevant to this article was reported.

\section{AUTHORS CONTRIBUTIONS}

Study design: M.F., A.F., M.B., K.K. Data acquisition: C.S., F.F., V.S., S.B., M.F., A.F., M.B., K.K. Data analysis: C.S., M.M., S.B., M.B., K.K. Writing of the manuscript: C.S., M.B., K.K.

\section{REFERENCES}

1. Ferlay J, Shin HR, Bray F, Forman D, Mathers C, Parkin DM. Estimates of worldwide burden of cancer in 2008: GLOBOCAN 2008. Int J Cancer 2010;127:2893-2917.

2. Forner A, Llovet JM, Bruix J. Hepatocellular carcinoma. Lancet 2012;379:1245-1255.

3. Altekruse SF, McGlynn KA, Reichman ME. Hepatocellular carcinoma incidence, mortality, and survival trends in the United States from 1975 to 2005. J ClinOncol 2009;27:1485-1491.

4. Siegel RL, Miller KD, Jemal A. Cancer statistics, 2016. CA Cancer J Clin 2016;66:7-30.

5. European Association for the Study of the Liver; European Organisation for Research and Treatment of Cancer. EASL-EORTC clinical practice guidelines: management of hepatocellular carcinoma. J Hepatol 2012;56:908-943.

6. Clavien PA, Petrowsky H, DeOliveira ML, Graf R. Strategies for safer liver surgery and partial liver transplantation. N Engl J Med 2007;356:1545-1559.

7. Chapman BC, Paniccia A, Hosokawa PW, et al. Impact of facility type and surgical volume on 10-year survival in patients undergoing hepatic resection for hepatocellular carcinoma. J Am Coll Surg 2017;224:362-372.

8. Berzigotti A, Reig M, Abraldes JG, Bosch J, Bruix J. Portal hypertension and the outcome of surgery for hepatocellular carcinoma in compensated cirrhosis: a systematic review and meta-analysis. Hepatology 2015;61:526-536.

9. Franchini M, Frattini F, Crestani S, Bonfanti C, Lippi G. von Willebrand factor and cancer: a renewed interest. Thromb Res 2013;131:290-292.

10. Marfia G, Navone SE, Fanizzi C, et al. Prognostic value of preoperative von Willebrand factor plasma levels in patients with Glioblastoma. Cancer Med 2016;5:1783-1790.

11. Yang X, Sun HJ, Li ZR, et al. Gastric cancer-associated enhancement of von Willebrand factor is regulated by vascular endothelial growth factor and related to disease severity. BMC Cancer 2015;15:80.

12. El Sherif NH, Narouz MF, Elkerdany TA, El Habashy SA. Von Willebrand factor and factor VIII levels in Egyptian children with newly diagnosed acute lymphoblastic leukemia in relation to peripheral blast cells and steroid therapy. J Pediatr Hematol Oncol
2014;36:518-523.

13. Homoncik M, Ferlitsch A, Ferenci P, et al. Short- and long-term effects of therapy with interferon-alpha and pegylated interferonalpha/ribavirin on platelet plug formation and von Willebrand factor release in patients with chronic hepatitis C. Aliment Pharmacol Ther 2005;21:49-55.

14. Krieger PM, Tamandl D, Herberger B, et al. Evaluation of chemotherapy-associated liver injury in patients with colorectal cancer liver metastases using indocyanine green clearance testing. Ann Surg Oncol 2011;18:1644-1650.

15. Kuntz HD, Meessen D, May B. Hepatic elimination of indocyanine green and antipyrine in patients with chronic liver disease. Med Welt 1982;33:909-910.

16. Ferlitsch A, Teml A, Reinisch W, et al. 6-thioguanine associated nodular regenerative hyperplasia in patients with inflammatory bowel disease may induce portal hypertension. Am J Gastroenterol 2007;102:2495-2503.

17. Reiberger T, Rutter K, Ferlitsch A, et al. Portal pressure predicts outcome and safety of antiviral therapy in cirrhotic patients with hepatitis C virus infection. Clin Gastroenterol Hepatol 2011;9:602608.e1.

18. Strasberg SM. Nomenclature of hepatic anatomy and resections: a review of the Brisbane 2000 system. J Hepatobiliary Pancreat Surg 2005;12:351-355.

19. Dindo D, Demartines N, Clavien PA. Classification of surgical complications: a new proposal with evaluation in a cohort of 6336 patients and results of a survey. Ann Surg 2004;240:205-213.

20. Edmondson HA, Steiner PE. Primary carcinoma of the liver: a study of 100 cases among 48,900 necropsies. Cancer 1954;7:462503.

21. Batts KP, Ludwig J. Chronic hepatitis: an update on terminology and reporting. Am J Surg Pathol 1995;19:1409-1417.

22. Llovet JM, Brú C, Bruix J. Prognosis of hepatocellular carcinoma: the BCLC staging classification. Semin Liver Dis 1999;19:329-338.

23. Llovet JM, Di Bisceglie AM, Bruix J, et al. Design and endpoints of clinical trials in hepatocellular carcinoma. J Natl Cancer Inst 2008;100:698-711.

24. Angermayr SA, Hellingwerf KJ. On the use of metabolic control analysis in the optimization of cyanobacterial biosolar cell factories. J Phys Chem B 2013;117:11169-11175.

25. Peck-Radosavljevic M, Angermayr B, Datz C, et al. Austrian consensus on the definition and treatment of portal hypertension and its complications (Billroth II). Wien Klin Wochenschr 2013;125:200-219.

26. Llovet JM, Fuster J, Bruix J. Intention-to-treat analysis of surgical treatment for early hepatocellular carcinoma: resection versus transplantation. Hepatology 1999;30:1434-1440.

27. Hidaka M, Takatsuki M, Soyama A, et al. Intraoperative portal venous pressure and long-term outcome after curative resection for hepatocellular carcinoma. Br J Surg 2012;99:1284-1289.

28. Ferlitsch M, Reiberger T, Hoke M, et al. von Willebrand factor as new noninvasive predictor of portal hypertension, decompensa- 
tion and mortality in patients with liver cirrhosis. Hepatology 2012;56:1439-1447.

29. La Mura V, Reverter JC, Flores-Arroyo A, et al. Von Willebrand factor levels predict clinical outcome in patients with cirrhosis and portal hypertension. Gut 2011;60:1133-1138.

30. Horvatits T, Drolz A, Roedl K, et al. Von Willebrand factor antigen for detection of hepatopulmonary syndrome in patients with cirrhosis. J Hepatol 2014;61:544-549.

31. Fan ST, Poon RT, Yeung C, et al. Outcome after partial hepatectomy for hepatocellular cancer within the Milan criteria. Br J Surg 2011;98:1292-1300.

32. Schellerer VS, Mueller-Bergh L, Merkel S, et al. The clinical value of von Willebrand factor in colorectal carcinomas. Am J Transl Res 2011;3:445-453.

33. Wang WS, Lin JK, Lin TC, et al. Plasma von Willebrand factor level as a prognostic indicator of patients with metastatic colorec- tal carcinoma. World J Gastroenterol 2005;11:2166-2170.

34. Zietek Z, Iwan-Zietek I, Paczulski R, Kotschy M, Wolski Z. von Willebrand factor antigen in blood plasma of patients with urinary bladder carcinoma. Thromb Res 1996;83:399-402.

35. Gadducci A, Baicchi U, Marrai R, et al. Pretreatment plasma levels of fibrinopeptide-A (FPA), D-dimer (DD), and von Willebrand factor (vWF) in patients with operable cervical cancer: influence of surgical-pathological stage, tumor size, histologic type, and lymph node status. Gynecol Oncol 1993;49:354-358.

36. Bauer AT, Suckau J, Frank K, et al. von Willebrand factor fibers promote cancer-associated platelet aggregation in malignant melanoma of mice and humans. Blood 2015;125:3153-3163.

37. Goertz L, Schneider SW, Desch A, et al. Heparins that block VEGFA-mediated von Willebrand factor fiber generation are potent inhibitors of hematogenous but not lymphatic metastasis. Oncotarget 2016;7:68527-68545. 